\title{
Certification of Approximate Roots of Exact Polynomial
}

\section{Systems}

At the 2016 AMS Fall Southeastern Sectional Meeting I will talk about my joint work with Jonathan Hauenstein and our student Tulay Ayyildiz Akoglu on the certification of solutions of polynomial systems.
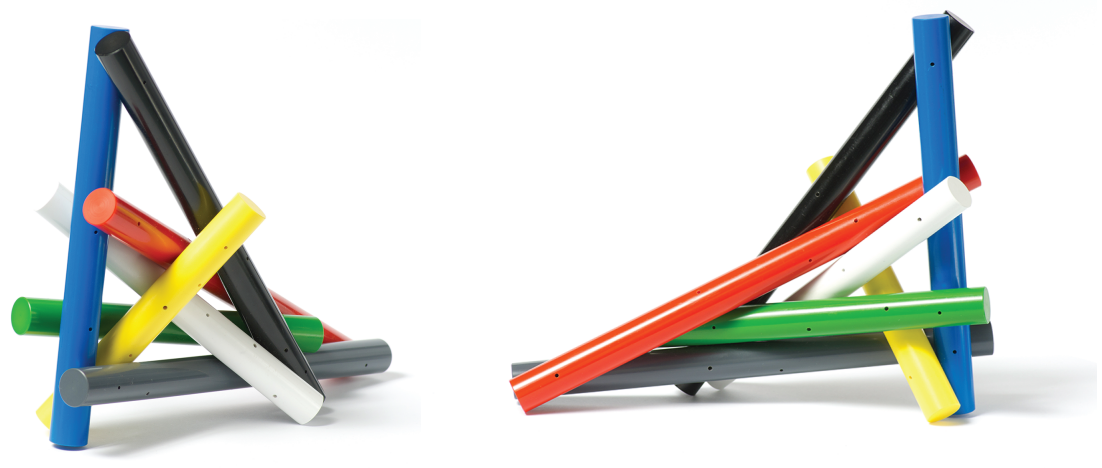

\section{Agnes Szanto}

I n the past fifty years, since the development of Buchberger's algorithm for computing Gröbner bases, symbolic computation has seen an explosion in development and applications for solving systems Lof polynomial equations. More recently, the combination of numerical analysis and algebraic geometry, yielding the field of numerical algebraic geometry, has produced new approaches and extended the limits of solving systems of polynomial equations. In general, these numerical methods are parallelizable and are producing solutions to large-scale systems. For example, the Bertini software package, a general-purpose numerical

Agnes Szanto is associate professor of mathematics at North Carolina State University. Her email address is aszanto@ncsu . edu.

Agnes was partially supported for this research by NSF Grant CCF-1217557.

For permission to reprint this article, please contact:

reprint-permission@ams.org.

DOI: http://dx.doi.org/10.1090/noti1440 solver for polynomial systems, has recently been used to solve a system of seventy quadratics in seventy variables [4]. An overview of numerical algebraic geometry with a focus on using the software Bertini can be found in [2].

Sophisticated numerical algebraic geometry techniques are also able to solve problems that were once thought to be purely symbolic in nature, corresponding to noncontinuous properties of the input polynomial system. Some of the surprising problems that can be handled using numerical methods include finding primary decompositions, the genus of irreducible curves, or intersection numbers of Chern classes. However, many of the results produced by numerical algebraic geometry are not certified, as they are generated using heuristic methods that relax noncontinuous properties into continuous ones. The aim of our work is to give certification techniques for these noncontinuous problems and to demonstrate that certificates can be computed with not too much extra work, given numerical data. 
As mentioned above, typically the output of numerical algebraic geometry computations are not certified, with one notable exception being the special case of nonsingular roots of well-constrained systems. Before explaining our contributions, let me describe $\alpha$-theory, one of the certification techniques from the extended body of literature for this special case.

Let $f: \mathbb{C}^{n} \rightarrow \mathbb{C}^{n}$ be a system of analytic functions and $\mathbf{z} \in \mathbb{C}^{n}$. Introduced by Smale in 1986, $\alpha$-theory guarantees quadratic convergence of the Newton iteration from $\mathbf{z}$ to a fixed point if an associated invariant $\alpha(f, \mathbf{z})$ is smaller than a universal constant. Thus, in the case of a nonsingular root of a well-constrained system, $\alpha$-theory gives a certificate that $\mathbf{z}$ is an approximate zero of $f$, i.e., that Newton's iteration starting from $\mathbf{z}$ quadratically converges to a root $\xi \in \mathbb{C}^{n}$ of $f$. Moreover, $\alpha$-theory also gives a bound on the distance of $\mathbf{z}$ from $\xi$ and certifies the uniqueness of the root within that distance. This last property allows $\alpha$-theory to certify that $\mathbf{z} \in \mathbb{R}^{n}$ approximates a real root of a real polynomial system, which is used in many applications.

The invariant $\alpha(f, \mathbf{z})$ depends on all derivatives of $f$ evaluated at $\mathbf{z}$. If $f$ is a polynomial system, an upper bound for $\alpha(f, \mathbf{z})$ can be efficiently evaluated, which is implemented in the software a]phaCertified [5]. A fascinating application is a computerized proof of a conjecture by John Edensor Littlewood, who asked the following question:

Is it possible in 3-space for seven infinite circular cylinders of unit radius each to touch all the others? Seven is the number suggested by constants.

Bozóki, Lee, and Rónyai [3] proved this conjecture by setting up a well-constrained polynomial system over $\mathbb{Z}$ with real roots corresponding to solutions of the Littlewood conjecture. They approximated the roots using a numerical homotopy continuation method and certified that some roots are real using a 1 phaCertified. A model of a cylinder arrangement solving Littlewood's conjecture that they found is shown in Figure 1.

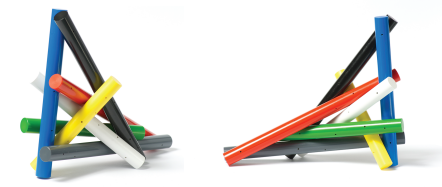

Figure 1. Bozóki, Lee, and Rónyai proved that seven cylinders can all touch each other.

Motivated by such applications, we turned our attention to polynomial systems that are overdetermined or have singular roots. Note that consistency of an overdetermined polynomial system is not a continuous property of the input, and similarly the multiplicity structure of a given root is destroyed by perturbations of the input system. Existing approaches to certify solutions to such systems permit small perturbations of the input system, transforming the problem into a continuous one, allowing the use of $\alpha$-theory or interval arithmetic or other continuous certification methods. However, for applications such as the computerized mathematical proof above, this is not sufficient: the polynomial system is given exactly with coefficients from $\mathbb{Z}$ or $\mathbb{Q}$, and we need to certify approximate zeroes of the given exact system without any perturbation.

Certifying the above noncontinuous properties posed completely new challenges and needed new ideas. Let me demonstrate the difficulties in a very simple example: Consider the overdetermined system $f: \mathbb{C} \rightarrow \mathbb{C}^{2}$ defined for any $a \in \mathbb{C}$ by

$$
f(x)=\left(\begin{array}{c}
x \\
x^{2}+a
\end{array}\right) .
$$

Dedieu and Shub developed an $\alpha$-theory for overdetermined systems using the so-called Gauss-Newton iteration, but this only certifies fixed points which may not be roots; e.g., $x=0$ is a fixed point of the Gauss-Newton iteration but not a root of $f$, unless $a=0$. In particular, roots and local minima of $\|f\|$ are indistinguishable when using continuous certification methods.

To overcome this problem, Hauenstein and Sottile considered using universal lower bounds for the minimum of positive polynomials over $\mathbb{Z}$ on a disk. They concluded that all known bounds were "too small to be practical." For example, the overdetermined system

$f_{1}:=x_{1}-\frac{1}{2}, f_{2}:=x_{2}-x_{1}^{2}, \ldots, f_{n}:=x_{n}-x_{n-1}^{2}, f_{n+1}:=x_{n}$

has no common roots, but the value of $f_{n+1}$ on the common root of $f_{1}, \ldots, f_{n}$ is doubly exponentially small in $n$. Since universal lower bounds have to cover these artificial cases, we cannot expect much improvement using universal bounds.

This led to the idea of constructing some individualized witness for our input polynomial system $f$ instead of using universal lower bounds that are often very pessimistic. In our approach we use the so-called Rational Univariate Representation (RUR) as our witness, and we reconstruct this exact representation from approximate data. The advantage of our approach, as we shall see, is the ability to terminate early in cases when the witness for our input instance is small.

The main idea of our symbolic-numeric method to certify overdetermined polynomial systems over $\mathbb{Q}$ is as follows: Consider $f=\left(f_{1}, \ldots, f_{m}\right) \in \mathbb{Q}\left[x_{1}, \ldots, x_{n}\right]^{m}$ for some $m>n$, and assume for now that all roots are isolated and nonsingular. Under these assumptions, the RUR for the common roots of $f$ exists, and the polynomials in the RUR also have rational coefficients. Thus we can compute them exactly, unlike the possibly irrational coordinates of the common roots of $f$. With the exact RUR, which is a well-constrained system of polynomials, we can use alphaCertified to certify that a given point is an approximate root for the RUR and thus for our original system $f$.

In principle, one can compute such an RUR using purely algebraic techniques, for example, by solving large linear systems corresponding to resultant or subresultant matrices. However, this purely symbolic method would again lead to running times close to the worst case complexity bounds. Instead, we propose a hybrid symbolic-numeric 
approach using interpolation on the approximate roots of $f$, rational number reconstruction, as well as exact univariate polynomial remaindering over $\mathbb{Q}$. Our hope is that our method will make the certification of roots of overdetermined systems practical for cases when the actual size of the RUR is significantly smaller than in the worst case.

The details of this hybrid symbolic-numeric method are described in our paper [1], which also contains detailed complexity analysis, demonstrating that in the case of successful certification the complexity is polynomial in the input plus the output size. This is a significant improvement over purely symbolic methods, which always have comparable complexity to our worst-case scenario when the certification fails. In other words, our method returns certification in many instances very quickly, but in some cases it terminates with failure.

Once we successfully certified nonsingular roots of overdetermined systems over $\mathbb{Q}$, we considered certifying isolated singular roots of rational polynomial systems. Due to the behavior of Newton's method near singular roots, standard techniques in $\alpha$-theory cannot be applied to certify such roots even if the polynomial system is well constrained. The key tool to handle such multiple roots is called deflation. Deflation techniques "regularize" the system, thereby creating a new polynomial system which has a simple root corresponding to the multiple root of the original system. In our work, we focus on using a determinantal form of the isosingular deflation, in which one simply adds new polynomials to the original system without introducing new variables. The new polynomials are certain subdeterminants of the Jacobian matrix of the polynomial system, constructed using exact information that one can obtain from a numerical approximation of the multiple root. In particular, if the original system had rational coefficients, the new polynomials which remove the multiplicity also have rational coefficients. Thus, this technique provides a reduction to the case of an overdetermined system over $\mathbb{Q}$ in the original set of variables that has a simple root.

We demonstrate our method on a common benchmark system, the Caprasse system, which has 24 regular roots and 8 roots of multiplicity 4 , namely,

$g=$

$$
\left[\begin{array}{c}
x_{1}^{3} x_{3}-4 x_{1}^{2} x_{2} x_{4}-4 x_{1} x_{2}^{2} x_{3}-2 x_{2}^{3} x_{4}-4 x_{1}^{2}-4 x_{1} x_{3}+10 x_{2}^{2}+10 x_{2} x_{4}-2 \\
x_{1} x_{3}^{3}-4 x_{1} x_{3} x_{4}^{2}-4 x_{2} x_{3}^{2} x_{4}-2 x_{2} x_{4}^{3}-4 x_{1} x_{3}+10 x_{2} x_{4}-4 x_{3}^{2}+10 x_{4}^{2}-2 \\
2 x_{1} x_{2} x_{4}+x_{2}^{2} x_{3}-2 x_{1}-x_{3} \\
x_{1} x_{4}^{2}+2 x_{2} x_{3} x_{4}-x_{1}-2 x_{3}
\end{array}\right]
$$

Since the system is well constrained, numerical approximations for the 24 regular roots can be certified using standard $\alpha$-theory. Here, we consider certifying the multiple roots. When evaluated at each of these multiple roots, the $4 \times 4$ Jacobian matrix $J_{g}=\left[\frac{\partial g_{i}}{\partial x_{j}}\right]_{i, j=1}^{4}$ has rank 2, with the lower right $2 \times 2$ block having full rank, which property can be obtained using approximate roots and numerical rank computations. Thus, we construct the new polynomial system $f$ by appending $g$ with the four $3 \times 3$ minors of $J_{g}$ containing the lower right block. Now $f$ is an overdetermined polynomial system over $\mathbb{Q}$ that completely deflates all 8 roots; i.e., they become nonsingular roots of $f$.

From the numerical approximations of the eight points that we computed using Bertini, we see that $T=$ $x_{1}-x_{2}+3 x_{3}-3 x_{4}$ separates the roots. Using interpolation on the numerical approximations of the roots correct to six digits we constructed the following exact RUR, which we certified to be correct using exact univariate polynomial remaindering:

$$
\begin{aligned}
q & =\left(T^{2}+4 / 3\right)\left(T^{2}+12\right)\left(T^{2}-16 T+76\right)\left(T^{2}+16 T+76\right), \\
r_{1} & =(224 / 3) T^{6}-(15232 / 3) T^{4}-6656 T^{2}+505856 / 3, \\
r_{2} & =-(80 / 3) T^{6}-1856 T^{4}-41216 T^{2}+97280 / 3, \\
r_{3} & =(160 / 3) T^{6}-2944 T^{4}-(363008 / 3) T^{2}-972800 / 3, \\
r_{4} & =(80 / 3) T^{6}+1856 T^{4}+41216 T^{2}-97280 / 3 .
\end{aligned}
$$

This RUR corresponds to a well-constrained system $\left.q, q^{\prime} x_{1} r_{1}, \ldots, q^{\prime} x_{4}-r_{4}\right) \in Q\left[T, x_{1}, x_{2}, x_{3}, x_{4}\right]^{5}$ defining the 8 roots, now with multiplicity one. Thus we can use alphaCertified to certify that a given point is an approximate root for the RUR and thus for our original systems $f$ and $g$.

\section{References}

[1] T. A. Akoglu, J. D. Hauenstein, and A. Szanto, Certifying solutions to overdetermined and singular polynomial systems over $\mathbb{Q}$, arXiv:1408.2721, 2014.

[2] D. J. BAtes, J. D. Hauenstein, A. J. Sommese, and C. W. WAMPLER, Numerically solving polynomial systems with Bertini, volume 25 of Software, Environments, and Tools, Society for Industrial and Applied Mathematics (SIAM), Philadelphia, PA, 2013. MR 3155500

[3] S. BOzÓKI, T.-L. LEE, and L. RÓNYAI, Seven mutually touching infinite cylinders. Comput. Geom. 48 (2015), no. 2, 87-93. MR 3260249

[4] J. HAUENSTEIN and J. MCCARTHY, Biologically-inspired linkage design: computing form from function. SIAM News 48 (2015), no. 8.

[5] J. D. Hauenstein and F. SotTile, Algorithm 921: alpha Certified: certifying solutions to polynomial systems, ACM Trans. Math. Software 38 (2012), no. 4, Art. ID 28, 20 pp. MR 2972672

\section{Credits}

Figure 1, courtesy of Peter Gal.

Photo of Agnes Szanto, courtesy of Scott Hellmann.

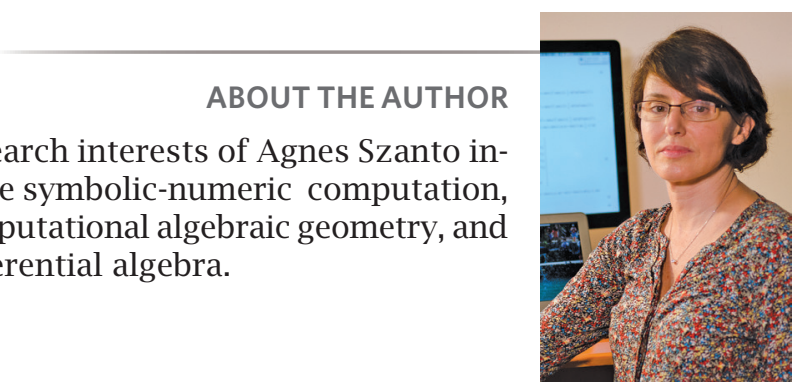

Agnes Szanto 